\title{
Performance of the HPC calorimeter in DELPHI
}

A. Algeri ${ }^{5)}$, C. Bastie ${ }^{3)}$,W. Bell ${ }^{3)}$, M. Boldini ${ }^{2)}$, R. Böhmer ${ }^{6)}$ W. Bonivento ${ }^{7}$, H. Burmeister ${ }^{3)}$, M. Calvi ${ }^{7)}$, K. Cankocak ${ }^{9)}$, A. Cattai ${ }^{3)}$, F. Cavallo ${ }^{2)}$, A. Chan ${ }^{1)}$, F. Chignoli ${ }^{7}$, S. Colilli ${ }^{8}$, R. Contri ${ }^{4}$, H.B. Crawley $\left.{ }^{1}\right)$, G. Crosetti ${ }^{4)}$, W. De Boer ${ }^{6}$, A. De $\mathrm{Min}^{7)}$, K. Doroba ${ }^{10)}$, D.M. Edsall ${ }^{1)}$, M. Feindt ${ }^{3)}$, A. Firestone ${ }^{1)}$, H. Furstenau ${ }^{3)}$, D. Gillespie ${ }^{3)}$, V. Giordano ${ }^{2)}$, M.S. Gorbics ${ }^{1)}$, L. Gorn ${ }^{1)}$, E. Graziani ${ }^{8}$, S.O. Holmgren ${ }^{9}$, K. Hultquist ${ }^{9)}$, M. Karlsson ${ }^{9)}$, B. König ${ }^{6)}$ W. Köhler ${ }^{6)}$ C. Kreuter ${ }^{6}$, J. Krolikowski ${ }^{10)}$, R. Jacobsson ${ }^{9}$, E. Johannson ${ }^{9)}$, J.W. Lamsa ${ }^{1)}$, R. Leoni ${ }^{7}$, M. Lewandowski ${ }^{10}$, T. Malgren ${ }^{9}$, T. Marszal ${ }^{10)}$, J. Martin ${ }^{3)}$, L. Maselli' ${ }^{2)}$, R. Mazza ${ }^{7)}$, R. McKay $^{1)}$, G. Meola ${ }^{4)}$, W.T. Meyer ${ }^{1)}$, T. Moa ${ }^{9)}$, A. Morelli ${ }^{4}$, P. Morettini ${ }^{4)}$, F.L. Navarria ${ }^{2)}$, P. Negri ${ }^{7}$, A. Passeri ${ }^{8)}$, A. Perrotta ${ }^{2)}$, G. Piana ${ }^{4)}$, O. Podobrin ${ }^{6}$, S. Ragazzi ${ }^{7}$, E.I. Rosemberg ${ }^{1)}$, U. Rossi ${ }^{2)}$, M. Sannino ${ }^{4)}$, S. Schael ${ }^{3)}$, A. Seitz ${ }^{6)}$, S. Sorrentino ${ }^{8)}$, R. Sosnowski10), E. Spiriti ${ }^{8)}$, S. Squarcia ${ }^{4)}$, C. Stanescu ${ }^{8)}$, M. Szczekowski ${ }^{10)}$, M. Szeptycka ${ }^{10)}$, P. Szymanski ${ }^{10)}$, T. Tabarelli de Fatis ${ }^{7)}$, L. Thollander ${ }^{9}$, W.D. Thomas ${ }^{1)}$, A. Tonazzo ${ }^{7}$, L. Tortora $^{8)}$, L. Traspedini ${ }^{4)}$, G. Valenti ${ }^{2)}$, M.R. Wayne ${ }^{1)}$, M. Wielers ${ }^{6}$

Presented by

\section{A. De Min}

\begin{abstract}
The performance of the High-density Projection Chamber (HPC), the barrel electromagnetic calorimeter of the DELPHI experiment, is described. The detector adopts the time projection technique in order to obtain exceptionally fine spatial granularity in the three coordinates $\left(\sim 2 \times 20 \mathrm{mrad}^{2}\right.$ in $\theta \times \phi$ with nine samplings along the shower axes), using a limited number of readout channels $(18,432)$. Among the various topics concerning the HPC construction and operation, major emphasis is given to the aspects related to the calibration in energy of the calorimeter, based mainly on the analysis of the detector response to ${ }^{83 m} \mathrm{Kr}$ decays, and to the treatment of ageing in the readout proportional counters.
\end{abstract}

Invited talk given at the IEEE-NSS94 Conference, Norfolk, Virginia, USA, November 1994. 
1) Ames Laboratory and Department of Physics, Iowa State University, Ames IA 50011, USA,

2) Dipartimento di Fisica, Università di Bologna and INFN, Via Irnerio 46, I-40126 Bologna, Italy,

3) CERN, CH-1211 Geneva 23, Switzerland,

4) Dipartimento di Fisica, Università di Genova and INFN, Via Dodecaneso 33, I-16146 Genova, Italy,

5) Department of High Energy Physics, University of Helsinki, Siltavuorenpenger 20 C, SF-00170 Helsinki 17, Finland,

6) Institut für Experimentelle Kernphysik, Universität Karlsruhe, Postfach 6980, D-7500 Karlsruhe 1, Germany,

7) Dipartimento di Fisica, Università di Milano and INFN, Via Celoria 16, I-20133 Milan, Italy,

8) Istituto Superiore di Sanità, INFN, Viale Regina Elena 299, I-00161 Rome, and Dipartimento di Fisica, Università di Roma II and INFN, Tor Vergata, I-00173 Rome, Italy,

9) Institute of Physics, University of Stockholm, Vanadisvägen 9, S-113 46, Stockholm, Sweden

10) Institute of Nuclear Studies and University of Warsaw, UL Hoza 69, PL-99681, Warsaw, Poland. 
The High-density Projection Chamber (HPC) is the barrel electromagnetic calorimeter of the DELPHI experiment at the CERN LEP collider. DELPHI [1] is a full solid angle multipurpose detector provided with high resolution tracking (Vertex Detector, TPC) in a 1.2 Tesla magnetic field, powerful particle identification (RICH) and finely granulated electromagnetic and hadronic calorimeters. LEP is presently running at $\sqrt{s} \sim m_{Z} \sim 91 \mathrm{GeV}$, where it benefits from the large resonant $e^{+} e^{-} \rightarrow Z \rightarrow f \bar{f}$ cross section.

The HPC represents one of the first large-scale applications of the time projection principle to gas sampling calorimetry. This technique provides extremely fine spatial granularity with an acceptable number of readout channels. The peculiar feature of such a detector is thus its capability to fully reconstruct the three-dimensional charge distribution produced by electromagnetic showers and interacting hadrons, which facilitates $e / \pi$ and $\gamma / \pi^{0}$ separation.

Among the various topics related to the HPC construction and operation, in this paper particular emphasis is placed on the procedure adopted for the detector calibration and the channel-to-channel equalization. In the HPC this is particularly delicate due to the inherent complexity of the detector design and to the large ageing affecting the readout chambers. The technique developed for the HPC calibration consists of adding small quantities of ${ }^{83 \mathrm{~m}} \mathrm{Kr}$ to the gas mixture flowing in the HPC volume and of analysing the detector response to the decay ${ }^{83 m} \mathrm{Kr} \rightarrow{ }^{83} \mathrm{Kr}$. Despite its simplicity, the method has proven to be extremely reliable in the channel-to-channel equalization and it provides an accurate monitoring of the detector response in the long run, which allows one to trace and compensate for the effects of ageing with great precision.

The layout of the paper is as follows. Section 2 describes the HPC design and performance. Section 3 discusses the effects induced by ageing on the HPC analog response. Section 4 is dedicated to the illustration of the calibration method based on ${ }^{83 m} \mathrm{Kr}$ and of its impact on the detector performance. In particular, the procedures adopted for the channel-to-channel equalization and for the monitoring and compensation of ageing effects are illustrated in detail. Finally, in Section 5, the results of studies aimed at anticipating the behaviour of the HPC calorimeter in the years to come are described. The conclusions are presented in Section 6.

\section{Detector Layout and Performance \\ 2.1 Construction and Operation}

The HPC is composed of 144 independent units (modules), which are arranged in a cylindrical structure centered around the beam interaction region (Figure 1). With an internal radius of $208 \mathrm{~cm}$ and a total length of $496 \mathrm{~cm}$ along the beam axis, the full detector covers the angular region between $43^{\circ}$ and $137^{\circ}$ in polar angle $(\theta)$.

Each module, trapezoidal in shape, consists of a time projection chamber in which 41 thin layers of lead have been inserted in order to act as absorber material for electromagnetic showers. The total depth of the calorimeter amounts to $17.5 X_{0}$.

The charge deposited by electromagnetic showers is sampled in the $8 \mathrm{~mm}$ wide gas gaps between the lead walls, which are filled with $\mathrm{Ar} / \mathrm{CH}_{4}$ (in the ratio 80/20). The charge is then drifted by an electric field towards one end of the module, where it is collected by proportional counters (gain $\sim 10^{4}$ ) and sampled with a frequency of $15 \mathrm{MHz}$. The sampling frequency determines a granularity of about $4 \mathrm{~mm}$ in the drift coordinate, which corresponds to $\sim 2 \mathrm{mrad}$ in the $\theta$ angle. In each module the cathode of the proportional counters is segmented into 128 pads. The pads, in three different sizes, provide a granular- 
ity varying between 10 and $30 \mathrm{mrad}$ in azimuthal angle $(\phi)$ as function of the depth and nine samplings along the radial coordinate (Figure 2). Taking into account the cathode segmentation, the number of readout channels in the HPC amounts to 18,432 (128 pads $\times 144$ modules).

The drift field is provided by the lead converter itself which is segmented into parallel wires. These are electrically isolated and connected to a voltage divider in order to act as an electric field cage. The wires, with trapezoidal section (1.8 $\mathrm{mm}$ wide), are glued to both sides of a fiberglass-epoxy support. The voltage gradient of $106 \mathrm{Volt} / \mathrm{cm}$ determines a drift velocity of $\sim 5.6 \mathrm{~cm} / \mu$ s for electrons in standard conditions.

In the narrow gas gaps the charge transmission is limited by transverse diffusion, once the electric field in the HPC and the magnetic field in DELPHI are precisely aligned. For this reason each HPC unit can be tilted by up to \pm 5 mrad in polar angle. Typical charge attenuation lengths observed in the $\mathrm{HPC}$ exceed $350 \mathrm{~cm}$.

The readout electronics consist of a shaping/amplifying stage, followed by fast digitization and zero-suppression. The shaping provides excellent tail suppression (less than $0.1 \%$ of peak height after $1 \mu \mathrm{s}$ ) and base line stability. The analog to digital conversion is provided by 8 -bit FADCs at $15 \mathrm{MHz}$ with bilinear conversion and effective dynamic range of $800: 1$. The efficient zero-suppression scheme reduces the average data transfer rate to only $\sim 14$ kbyte per hadronic interaction at LEP.

For fast triggering purposes, a plane of scintillators is inserted into one of the sampling gas gaps close to the shower maximum (behind $4.5 X_{0}$ ). The readout uses $4-6 \mathrm{~m}$ long plastic fibers, which are connected to photomultiplier tubes mounted on the external surface of DELPHI.

\subsection{Performance}

A clear demonstration of the HPC unique granularity is shown in Figure 3, where the reconstructed transverse charge distribution of a single photon is compared with that of two partially overlapping photons from a $\pi^{0}$ decay. The detailed analysis of the shower internal structure allows for efficient $\pi^{0} / \gamma$ separation even at relatively high energies. As an example, the inclusive cross section for $\pi^{0}$ production in $Z \rightarrow q \bar{q}$ decays can be measured in DELPHI for $\pi^{0}$ energies up to $\sim 20 \mathrm{GeV}[2]$.

In the HPC the electron identification is based mainly on the analysis of the longitudinal shower profile [3]. When combined with the information provided by the tracking detectors $(\mathrm{E} / \mathrm{p}, \mathrm{dE} / \mathrm{dx})$, the misidentification probability for electrons with momentum p $>3 \mathrm{GeV} / \mathrm{c}$ in $Z \rightarrow q \bar{q}$ events is less than $1.6 \%$ with $85 \%$ efficiency and less than $0.1 \%$ with $60 \%$ efficiency.

The spatial resolution has been measured in the data for high energy electrons by comparing the particle impact point as reconstructed by the HPC alone and by extrapolating the particle trajectory from the tracking detectors. In the drift coordinate the resolution varies between $1.3 \mathrm{~mm}$ and $3.1 \mathrm{~mm}$ as a function of $\theta$ and it is limited mainly by local instabilities in the drift velocity. In azimuthal angle the resolution has proven to be better than $10 \mathrm{mrad}$.

The energy resolution of the HPC is measured by analysing Bhabha $\left(e^{+} e^{-} \rightarrow e^{+} e^{-}\right)$ events, which are abundantly produced in Z decays and whose energy is constrained by kinematics to be equal to the (precisely known) beam energy. The observed resolution is $(6.4 \pm 0.2) \%$ at $45 \mathrm{GeV}$. Bhabha events also provide the reference energy scale for the absolute calibration of the calorimeter. More details about the detector equalization and calibration are given in Section 4. 
The HPC readout proportional counters are affected by a large ageing, which in average determines a relative amplitude loss of $10-15 \%$ per year in the analog response. As discussed in the following, the large amplitude loss is a consequence of both the intrinsically high specific ageing affecting the readout chambers and the large amount of charge induced in the chambers by the natural $\alpha$ radioactivity of the lead converter.

The specific ageing $(R)$ is usually defined as the relative amplitude loss in the chamber analog response per linear density of charge collected at the anode wire [4][5]:

$$
R=\left(\frac{\Delta A}{A}\right)\left(\frac{\Delta Q}{l}\right)^{-1}
$$

The amplitude loss in a period is thus obtained by multiplying $R$ by the integrated charge per unit length. The average specific ageing observed in the HPC chambers in the years 1990 to 1993 is shown in Table 1. The measured values show approximate stability in time, although different chamber gains were adopted in the four years. More accurate analyses, however, show a non-negligible dependence of the specific ageing on the chamber gain and the charge collection rate [6][7][8]. It should be stressed that the values reported in Table 1 $\left(R \sim(1.5-1.8) \times 10^{3} \mathrm{~cm} \mathrm{Coulomb}^{-1}\right)$ are indeed several orders of magnitude larger than those typically encountered in proportional counters, which range in the interval $R=\left(10^{-2}-10^{0}\right) \mathrm{cm} \mathrm{Coulomb}^{-1}$ [4][5]. Despite the large number of tests performed on the HPC, the cause of such a large ageing speed has never been unambiguously identified and no fully satisfactory solution has ever been found.

\begin{tabular}{|c|c|c|c|c|}
\hline Year & $\begin{array}{c}<\mathrm{I}> \\
{[\mathrm{nA}]}\end{array}$ & $\begin{array}{c}<\text { Specific ageing }> \\
{\left[\mathrm{cm} C o u l o m b^{-1}\right]}\end{array}$ & $\begin{array}{c}<\text { Ageing }> \\
{[\% / \text { day }]}\end{array}$ & $\begin{array}{c}\text { Loss per } \\
\text { year [\%] }\end{array}$ \\
\hline \hline 1990 & 58 & $(1.7 \pm 0.2) \cdot 10^{3}$ & $0.43 \pm 0.04$ & $\sim 20$ \\
1991 & 28 & $(1.8 \pm 0.2) \cdot 10^{3}$ & $0.22 \pm 0.02$ & $\sim 15$ \\
1992 & 34 & $(1.5 \pm 0.1) \cdot 10^{3}$ & $0.23 \pm 0.02$ & $\sim 15$ \\
1993 & 22 & $(1.8 \pm 0.2) \cdot 10^{3}$ & $0.18 \pm 0.02$ & $\sim 12$ \\
\hline
\end{tabular}

Table 1: Average values concerning the HPC chamber ageing measured with ${ }^{83 m} \mathrm{Kr}$ from 1990 to 1993: average chamber current $(<I>)$, specific ageing, relative amplitude loss per day and per year. The amplitude loss per year is the product of the loss per day and the length of the data taking period in each year.

The second factor determining the amplitude loss is the total amount of charge integrated by the readout chambers. In the HPC large quantities of ionization charge are deposited in the drift volume by $\alpha$ particles emitted by the lead converter $\left(\sim 70 \mathrm{~m}^{-2} \mathrm{~s}^{-1}\right)$. The effect is amplified by the peculiar "wire" structure of the converter which is characterized by a large surface exposure $\left(\sim 150 \mathrm{~m}^{2}\right.$ per module $)$ to the gas and induces a continuous current of a few tens of $\mathrm{nA}$ at the anode. The current amplitude varies from module to module due to the different origin of the lead samples and to the different drift characteristics; as a consequence different ageing amplitude losses are induced in each of the 144 HPC units. It should be noted that the current generated by $\alpha$ particles in the HPC is several orders of magnitude larger than that induced by $\mathrm{Z}$ decay products at LEP luminosities.

As shown in table 1 the average current drawn in the HPC chambers was progressively reduced during the years by modifying the chamber gain. In fact, by optimizing 
the working conditions, the absolute amplitude loss in one year of operation could be reduced from $\sim 20 \%$ to $\sim 12 \%$ in the period from 1990 to 1993. In particular the last decrease in the average current from 1992 to 1993 was achieved by reducing the chamber gain by a factor of two, which was compensated for by an equal increase in the gain of the amplifying stage of the readout electronics [9].

Due to these large effects, the use of a fast and reliable equalization procedure is essential for the complete control of the detector systematics. As discussed in more detail in the following section, this is achieved by means of periodic ${ }^{83 m} \mathrm{Kr}$ calibration runs.

\section{$4 \quad$ Energy Calibration}

\subsection{Channel-to-Channel Equalization}

The equalization of the 18,432 readout channels is obtained by analysing the detector response to ${ }^{83 m} \mathrm{Kr}$ decay. ${ }^{1)}$ Krypton gas is generated by a ${ }^{83} \mathrm{Rb}$ source placed in a by-pass of the gas supply system as shown in Figure 4 . The $\mathrm{Rb} \rightarrow \mathrm{Kr}$ transition leaves the $\mathrm{Kr}$ in an excited metastable state at $41.5 \mathrm{keV}\left({ }^{83 \mathrm{~m}} \mathrm{Kr}\right)$. While the gas is uniformly diffusing inside the HPC volume $\left(\sim 25,0001\right.$ in total) ${ }^{83 m} \mathrm{Kr}$ decays to the ground state $(\tau=1.86 \mathrm{~h})$ by emitting two photons of energies $32.1 \mathrm{keV}$ and $9.4 \mathrm{keV}$ in cascade.

\begin{tabular}{|c|l|c|}
\hline $\begin{array}{c}\text { Transition } \\
\text { energy }\end{array}$ & Decay mode & $\begin{array}{c}\text { Branching } \\
\text { ratio }\end{array}$ \\
\hline \hline $32.1 \mathrm{keV}$ & $\mathrm{e}(30 \mathrm{keV})+\mathrm{e}(2 \mathrm{keV})$ & $76 \%$ \\
& $\mathrm{e}(18 \mathrm{keV})+\mathrm{e}(10 \mathrm{keV})+2 \mathrm{e}(2 \mathrm{keV})$ & $9 \%$ \\
& $\mathrm{e}(18 \mathrm{keV})+X(12 \mathrm{keV})+\mathrm{e}(2 \mathrm{keV})$ & $15 \%$ \\
\hline \hline $9.4 \mathrm{keV}$ & $\mathrm{e}(7.6 \mathrm{keV})+\mathrm{e}(1.8 \mathrm{keV})$ & $95 \%$ \\
& $\gamma$ & $5 \%$ \\
\hline
\end{tabular}

Table 2: Decay channels of the ${ }^{83 m} \mathrm{Kr}$ isotope.

The high probabilities of internal conversion and Auger emission determine the decay pattern shown in table 2 : in about $80 \%$ of cases the energy is fully transferred to electrons, while in the remaining cases about $25 \%$ of the energy is emitted as electromagnetic radiation (9.4 keV $\gamma$-ray or $12 \mathrm{keV} \mathrm{X}$-ray) and escapes detection. The low energy electrons produced in ${ }^{83 m} \mathrm{Kr}$ decay curl in the DELPHI magnetic field and release all their energy within one drift gas gap, so that the charge is normally collected by a single cathode pad. In each pad, the ${ }^{83 m} \mathrm{Kr}$ energy spectrum consists of a full energy peak at $41.5 \mathrm{keV}$ and an escape peak around $30 \mathrm{keV}$. The two lines contributing to the escape peak cannot be resolved by the detector energy resolution (see for example Figure 6).

The readout channels are equalized with reference to the full energy $(41.5 \mathrm{keV})$ peak. The statistical precision of the equalization has been estimated from the comparison of the equalization coefficients obtained in two independent calibrations, as shown in Figure 5. Since two calibrations statistically comparable have been used, the width of the distribution shown in Figure 5 divided by $\sqrt{2}$ gives a rough estimate of the statistical precision of the equalization. The three distributions reported correspond to pads of large, medium and small size, whose areas are in the ratio 8:2:1. As expected a better equalization is obtained on large pads and the difference in precision is of a statistical nature. In any case the equalization precision is always better than $5 \%$.

1) The method was first suggested by the ALEPH Collaboration [10]. 
The effect of the spread in the pad-to-pad equalization on the HPC response to electromagnetic showers is expected to scale as $1 / \sqrt{N}$, where $N$ is the average number of pads hit by an electromagnetic shower. At Bhabha energies one has $N \sim 30$. Thus, assuming a pessimistic spread of about $5 \%$ in the pad equalization, a systematic contribution below $1 \%$ to the energy resolution of the HPC at $45 \mathrm{GeV}$ is estimated.

The equalization has to be repeated periodically, at intervals of about one year, as the spread in the pad equalization coefficients was observed to increase slightly with time [11]. These long term instabilities can be understood as caused by local fluctuations in the ageing speed.

\subsection{Ageing Monitoring and Compensation}

Once all the readout channels are properly equalized, the response of the detector to electromagnetic showers can be evaluated. As already mentioned, at LEP this is normally achieved by analysing Bhabha events. However in the case of the HPC the large ageing speed affecting the readout chambers requires the use of an independent method of monitoring the amplitude loss as function of time in each of the 144 units. Only after correcting for the amplitude loss, can the absolute scale be fixed using Bhabha events. Again ${ }^{83 m} \mathrm{Kr}$ calibrations provide the necessary tool to estimate the amplitude loss as function of time in each individual module.

In this case the module response is defined as the sum of the ${ }^{83 m} \mathrm{Kr}$ spectra observed in each of the 128 readout pads, after proper pad equalization. In typical calibration runs some $10^{3}$ events per module are collected, which ensures a statistical accuracy better than $1 \%$ on the peak position (Figure 6 ). The module calibration precision is then limited by the systematic effects related to the corrections for pressure and temperature fluctuations (with uncertainties $\sigma_{P} \sim \sigma_{T} \sim \pm 1 \%$ on the gas gain) and instabilities in the high voltage supply system $\left(\sigma_{H V} \sim 0.5 \%\right)$.

During one year, monthly calibrations provide a relative accuracy of $\pm 2 \%$ on the measurement of the ageing speed in each module. As the maximum amplitude loss registered is normally less than $20 \%$, the overall uncertainty induced by ageing on the absolute detector calibration turns out to be much less than $1 \%$.

It should be noted that because of differences between the ionization process characterizing ${ }^{83 m} \mathrm{Kr}$ decays and genuine electromagnetic showers, the absolute detector calibration cannot be derived from the ${ }^{83 m} \mathrm{Kr}$ analysis: while in the latter case the charge is released over the whole gap between two lead layers, in the former it is confined to a small region compared to the gap dimensions. It follows that the ${ }^{83 m} \mathrm{Kr}$ peak position is almost insensitive to the variations of the drift channel transparency from module to module, because the ionization charge is either completely lost or completely collected, which is definitely not true for electromagnetic showers. As a consequence ${ }^{83 m} \mathrm{Kr}$ calibrations can only provide a measurement of the relative amplitude loss as function of time.

Indeed, Figure 7 shows that ageing corrections deduced from the ${ }^{83 m} \mathrm{Kr}$ data can reliably compensate for the loss in the detector response observed in Bhabha events. Not only is the amplitude loss perfectly corrected for, but also the spread in the data points is decreased when the ${ }^{83 m} \mathrm{Kr}$ module-dependent corrections are implemented. The residual dispersion around the average value is compatible with the statistical error. This result guarantees the possibility to reliably equalize the HPC modules also at LEP200, where Bhabha events will not be available with such large statistics as on peak of the Z resonance. The summary of the average HPC behaviour in the years 1990 to 1993, as monitored by means of ${ }^{83 m} \mathrm{Kr}$ calibrations, is given in table 1. 


\subsection{Hardware equalization of the modules}

Software off line corrections do not avoid the occurrence of time-dependent threshold effects at low energy, which are induced by the progressive decrease of the chamber analog response. In order to reduce such effects, once per year hardware equalizations are taken on the HPC modules. These consist of compensating for the ageing amplitude loss accumulated in the previous year by tuning the chamber voltage independently in each of the 144 modules. Normally hardware equalizations are taken at the beginning of the data taking period, while, during the year, the ageing amplitude loss is studied with ${ }^{83 m} \mathrm{Kr}$ and is compensated for with off line corrections. In one year's time the dispersion in the module response has never exceeded $7-8 \%$.

As an example, the distribution of the high voltage settings adopted for the 1993 data taking is shown in Figure 8. The narrow peak on the left of the figure corresponds to 14 modules with new or rewired chambers, which were installed in the HPC during the LEP shutdown at the end of 1992 [9], to replace some of the most aged chambers. The difference between their high voltage $(1130 \mathrm{~V})$ and the average high voltage of the other modules $(1192 \mathrm{~V})$, translated in term of gas multiplication, gives a rough estimate of the average amplitude loss of the HPC calorimeter since the beginning of its operation, which turns out to be about $50 \%$.

The data also show that the spread in the high voltage values needed to equalize the $\mathrm{HPC}$ is increasing with time, as a consequence of the different ageing speed characterizing the modules. The typical RMS dispersion of about $20-30 \mathrm{~V}$ would result in a dispersion of about $25 \%$ in the response of the modules, if all of them were supplied with the same high voltage. For comparison, the 14 non-aged modules, installed at the end of 1992 and supplied with the same high voltage, show a dispersion in the analog response below $4 \%$. The data demonstrate that hardware equalizations are possible with an accuracy better than $3.5 \%$. Thus the dispersion after hardware equalizations is of the same order of that shown by new chambers at the same high voltage. This demonstrates that the procedure is well suited to keep under control the systematic effects related to variations in the analog response produced by ageing.

\section{$5 \quad$ Long Term Behaviour}

In order to have complete control over the HPC performance until the end of the LEP era (presently forseen for the year 2000), one also needs to investigate whether the ageing can introduce secondary effects in the detector response which can manifest themselves in the long run, like deviation from linearity, deterioration of the energy resolution, chamber breakdown, etc..

In order to anticipate the detector performance in the years to come, the most aged module (denominated C4) was extracted from the HPC in winter 1992/93 and was replaced by a spare module [9]. The module was particularly aged as it had been used in beam tests during 1988, that is before the start up of LEP. After the extraction, ageing was forced on the $\mathrm{C} 4$ module by increasing slightly the chamber gain, which in turn amplified the current produced by $\alpha$ particles, and by taking frequent ${ }^{83 \mathrm{~m}} \mathrm{Kr}$ calibration runs at moderate rate.

The module performance was then periodically monitored by means of test beams (twice per year), which were essentially dedicated to the measurement of energy resolution and linearity of response. The last test took place in October 1994, when the ageing status of the $\mathrm{C} 4$ module corresponded to that expected for the HPC in average in the year 2011. The main results of the test are summarized in Figure 9, where the observed 
energy resolution and linearity of response have been plotted as function of the beam energy. In particular, no deviation from linearity is observed for energies up to $50 \mathrm{GeV}$. This guarantees that, even in these extreme conditions, the method of fixing the absolute calibration scale with Bhabha events at $45 \mathrm{GeV}$ is reliable. ${ }^{2)}$ Furthermore, Figure 9 shows that the energy resolution does not seem to be significantly affected by ageing, as the resolution measured in 1994 on the $\mathrm{C} 4$ module is comparable to that observed on the same module in 1988, when the readout chambers were new.

\section{Conclusions}

The extremely fine granularity provided by the time projection technique makes the HPC a unique detector in the class of the large scale electromagnetic calorimeters.

The large amount of data collected in the first four years of operation shows that the detector has been successfully operated and has performed according to its design parameters.

Long dedicated studies have shown that the large ageing speed observed in the readout proportional counters does not significantly affect the detector performance and reliability until well after the end of the expected LEP data taking period.

Much credit for the understanding of the detector systematics and the exploiting of its performance goes to the use of ${ }^{83 \mathrm{~m}} \mathrm{Kr}$ gas in the calibration procedures, which not only provides fast and reliable channel-to-channel equalization but also allows for accurate monitoring and compensation of the progressive amplitude loss caused by ageing.

\section{References}

[1] DELPHI Collaboration, P. Aarnio et al., Nucl. Instr. and Meth. A303 (1991) 233.

[2] M. Feindt et al., Measurement of Inclusive $\pi^{0}$ Production in $Z^{0}$ decays, DELPHI Internal Note 94-81 PHYS 396 (1994), DELPHI contribution to the 1994 International Conference in High Energy Physics, Glasgow, UK, July 1994.

[3] C. Kreuter, Longitudinal Shower Development in the DELPHI Electromagnetic Calorirmeter HPC, IEKP-KA/93-9 (1993).

[4] J. Va'vra, Review of Wire Chamber Ageing, in Workshop on Radiation Damage to Wire Chambers, J.A. Kadyk ed., Berkeley, USA, 1986.

[5] J.A. Kadyk, Wire Chamber Ageing, Nucl. Instr. and Meth. A300 (1991) 436.

[6] A. Algeri et al., DELPHI Internal Note 92-103 CAL 100 (1992).

[7] A. Algeri et al., Nucl. Instr. and Meth. A338 (1994) 348.

[8] A. Cattai et al., Monitoring of the HPC by means of Radioactive Gas Calibrations, DELPHI Internal Note 93-129 CAL 108 (1993).

[9] A. Algeri et al., HPC Intervention during the 1992/93 Shutdown, DELPHI Internal Note 93-145 CAL 110 (1993).

[10] ALEPH Collaboration, D. Decamp et al., Nucl. Instr. and Meth. A294 (1990) 121.

[11] A. Cattai et al., Equalization of the Readout Channels of the HPC by means of Radioactive Gas, DELPHI Internal Note 93-115 CAL 105 (1993).

2) The deviation from linearity visible above $60 \mathrm{GeV}$ is due to leakage and gas saturation at high energy and is not induced by ageing. 


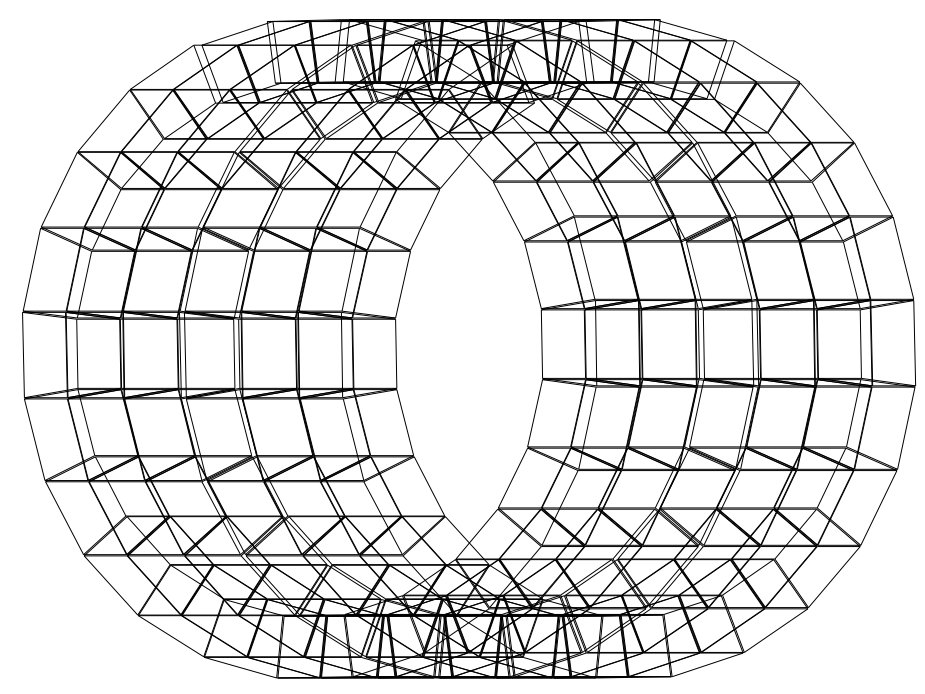

Figure 1: The disposition of the 144 modules in the HPC cylindrical structure.

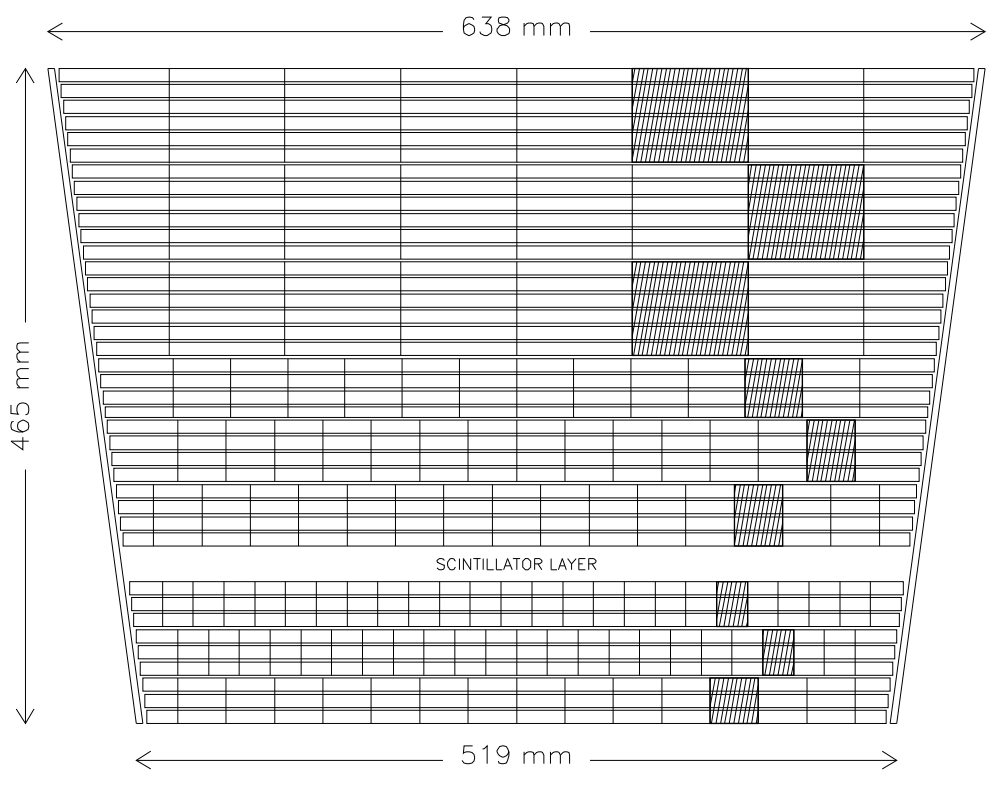

Figure 2: The cathode pad segmentation in the HPC readout proportional counters. 

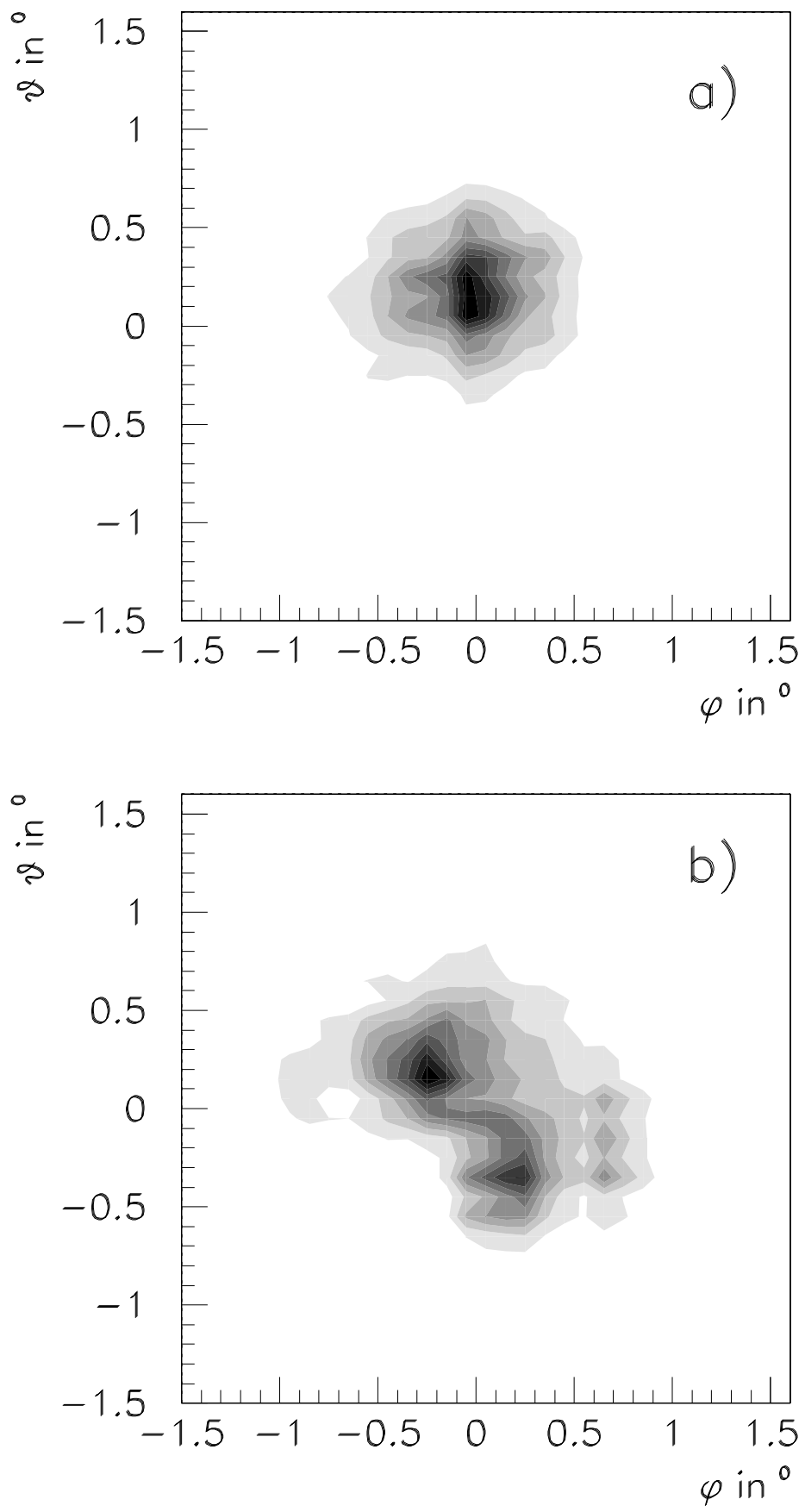

Figure 3: The transversal charge distribution reconstructed by the HPC for a single photon (a) and two partially overlapping photons from $\pi^{0}$ decay (b). 


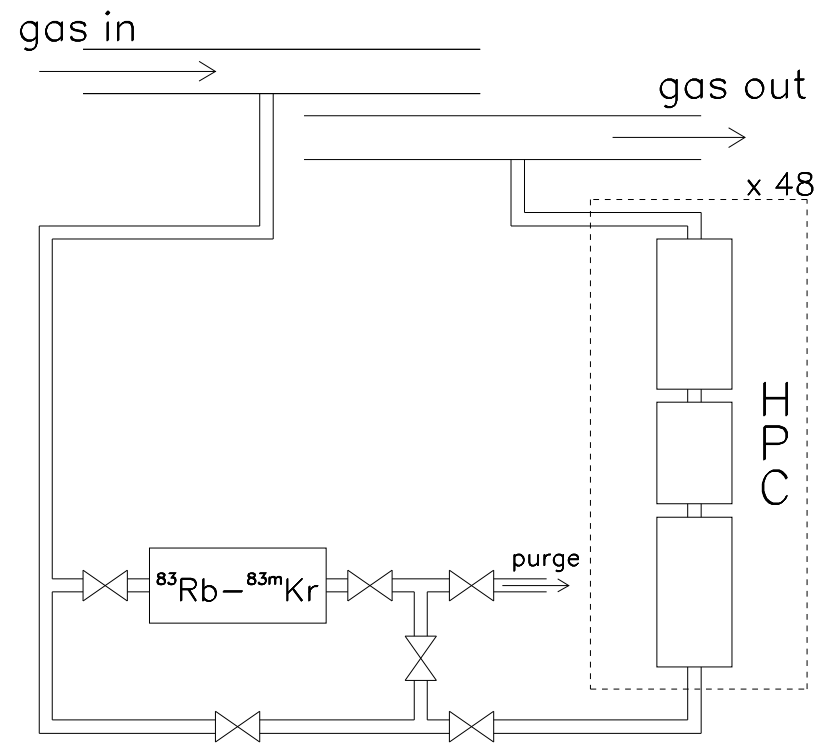

Figure 4: The ${ }^{83} \mathrm{Rb}_{-}{ }^{83 m} \mathrm{Kr}$ source is located in a by-pass of the input gas line, which is opened during calibration runs. The time needed to the radioactive gas to reach the HPC is much less than its decay time.
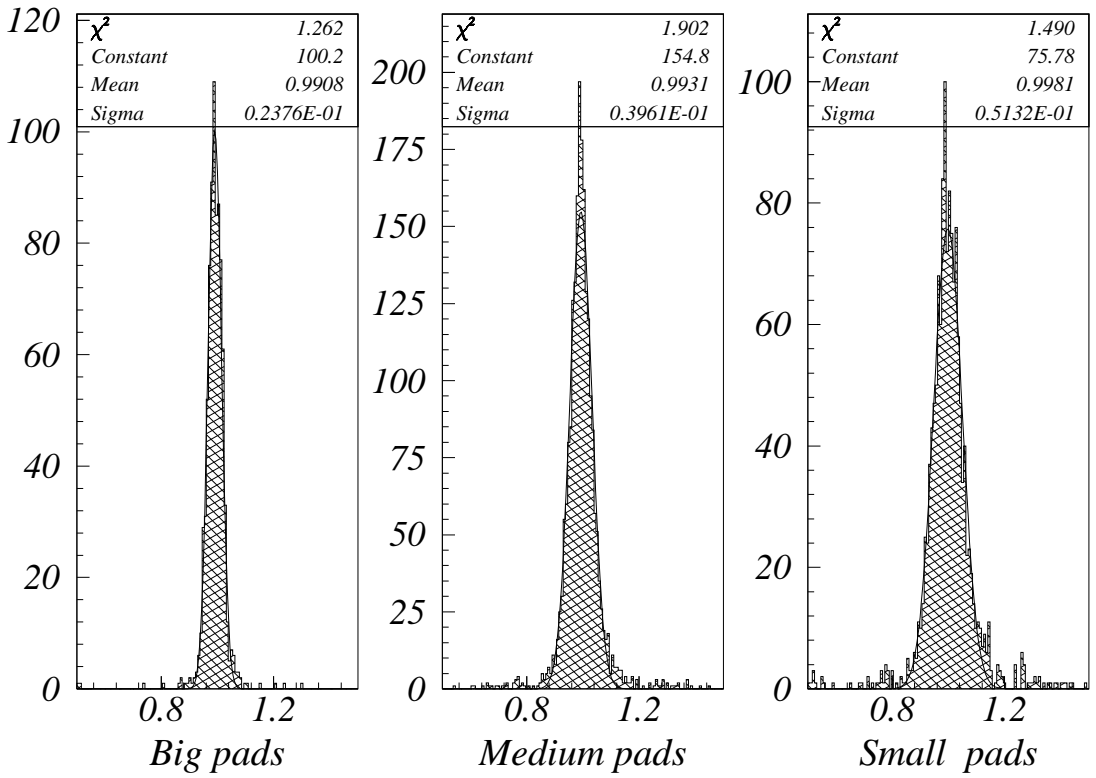

Figure 5: Ratio between equalization coefficents determined in two independent and statistically comparable calibration runs. 


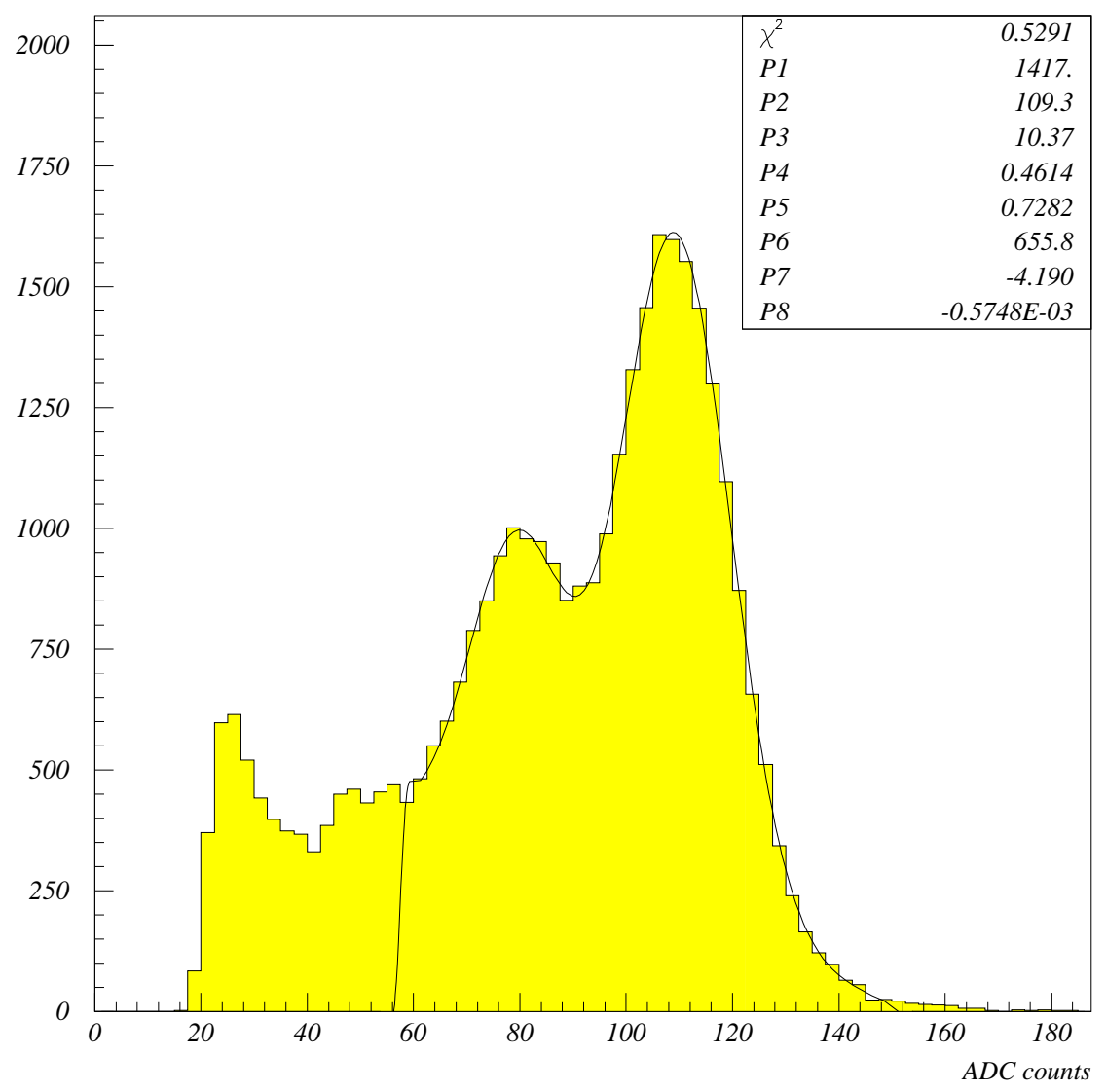

Figure 6: A typical ${ }^{83 m} \mathrm{Kr}$ spectrum observed in a HPC module. The global module spectrum is obtained by summing up the spectra of the 128 readout pads. The fit is based on two gaussian curves added to a quadratic background. 

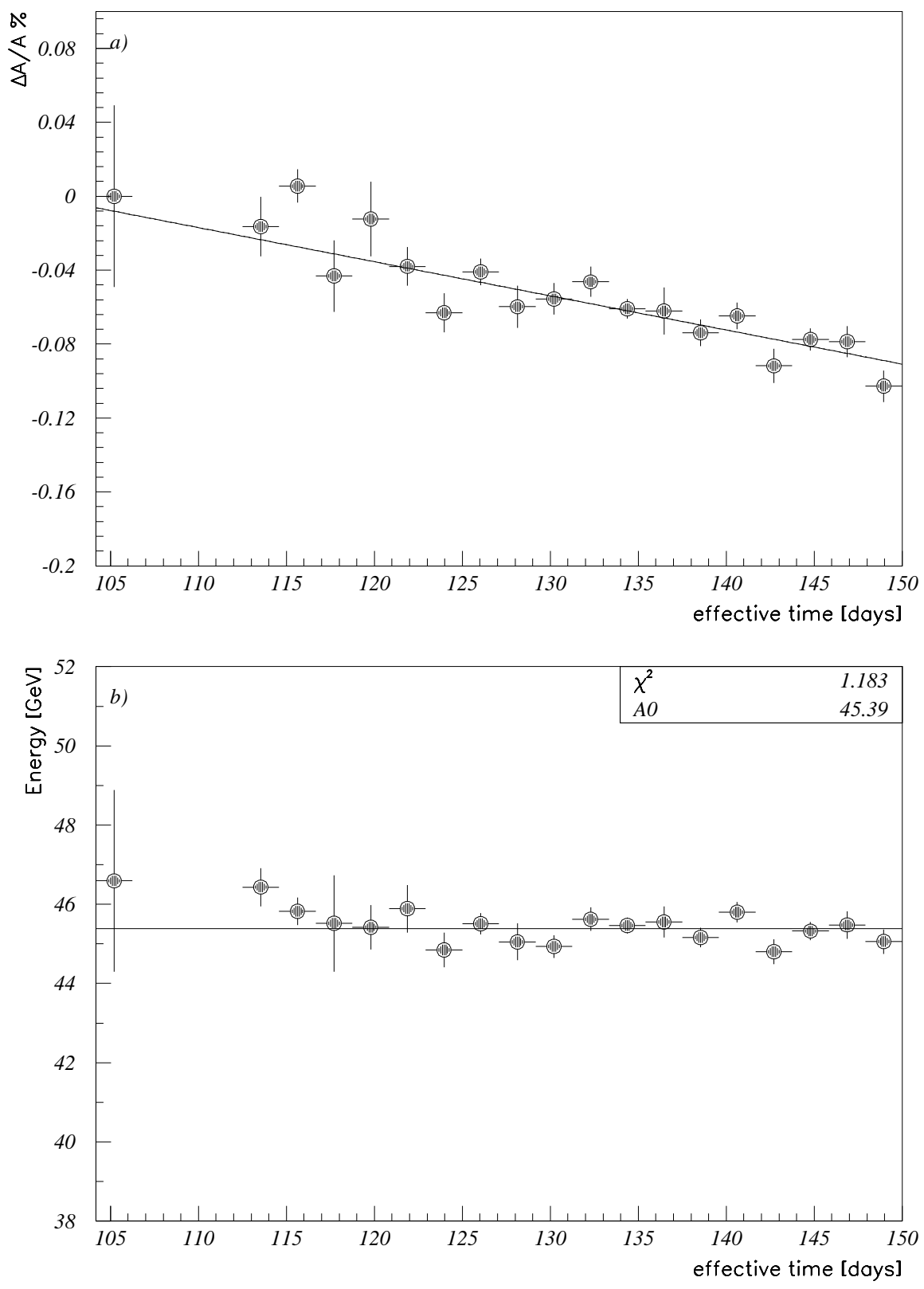

Figure 7: a) Decrease of the HPC analog response to $45 \mathrm{GeV}$ Bhabha events measured in the period May-August 1992; b) reconstructed energy after implementing the module-dependent ageing corrections determined from ${ }^{83 m} \mathrm{Kr}$ calibrations. 


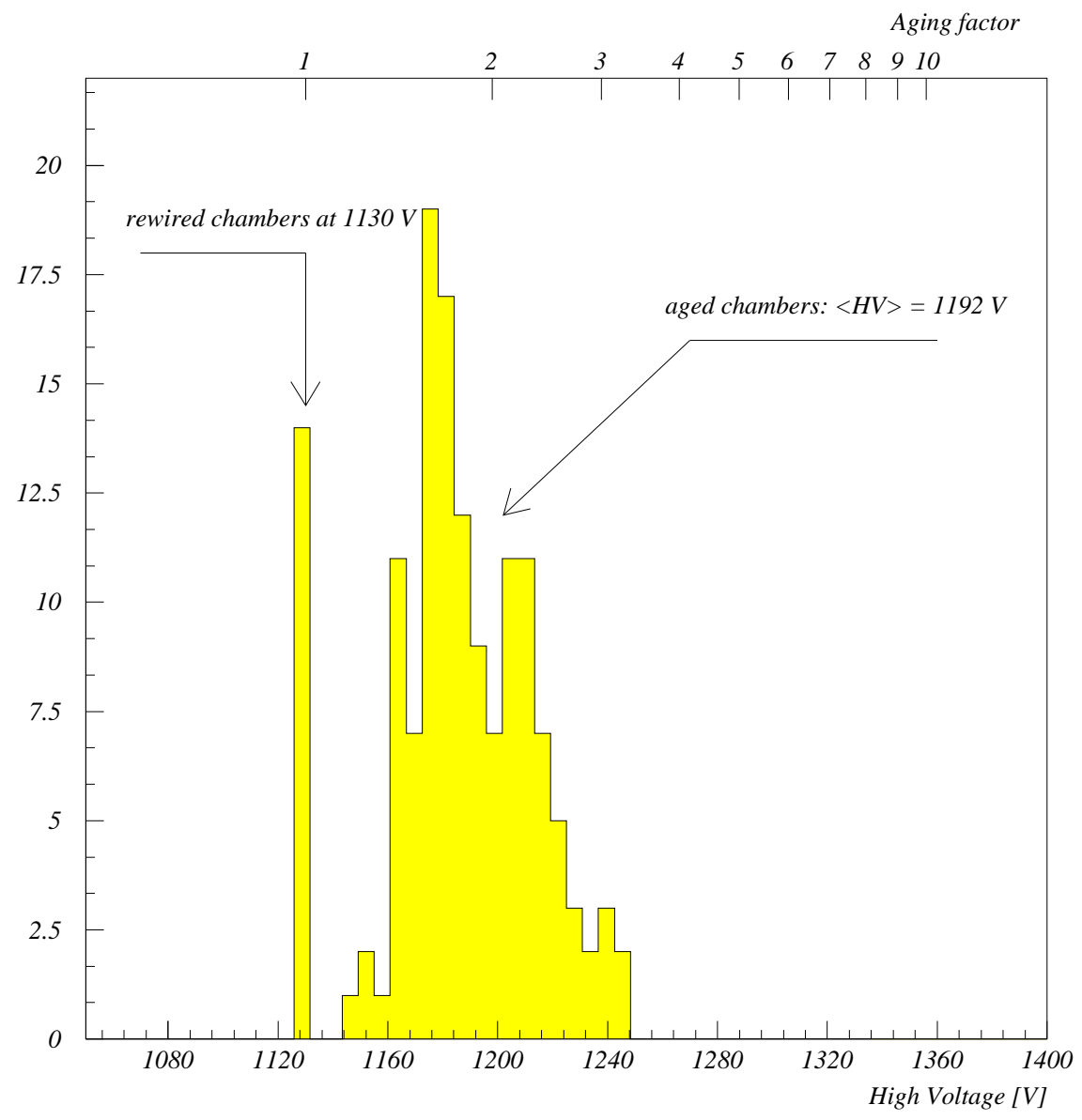

Figure 8: Distribution of the high voltage values needed to hardware-equalize the analog response of the HPC modules at the beginning of 1993 data taking. The peak at $1130 \mathrm{~V}$ is due to 14 modules with rewired or new readout chambers. The upper scale gives the ageing factor of the module, defined as the ratio between the analog response of a new chamber and that presently observed in each chamber at the same high voltage. 


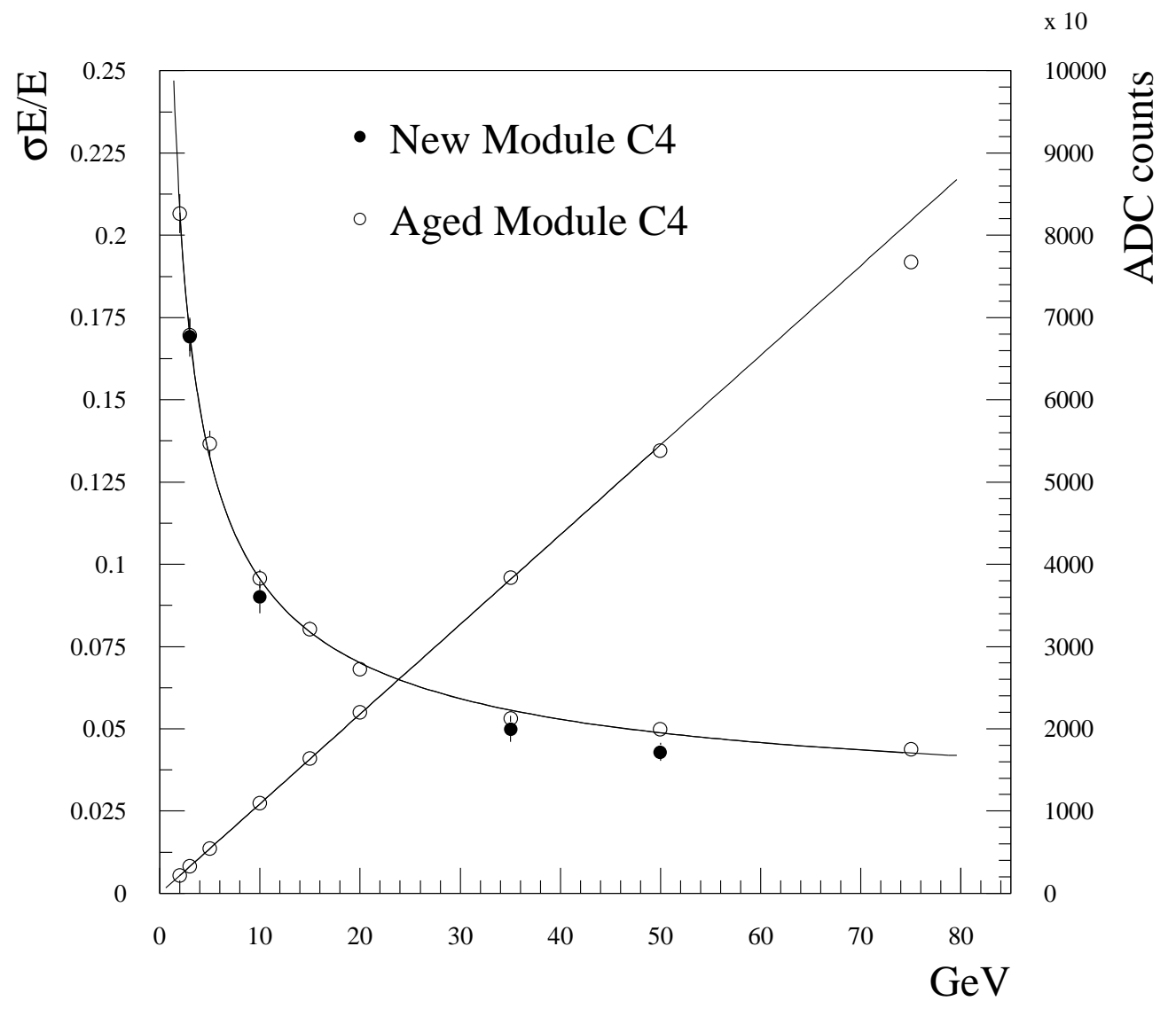

Figure 9: The empty dots show the linearity of response (right scale) and the energy resolution (left scale) measured on the aged module C4 in 1994. The full dots show the resolution observed in the same module in 1988, when the readout chambers were new. 